This item was submitted to Loughborough's Research Repository by the author.

Items in Figshare are protected by copyright, with all rights reserved, unless otherwise indicated.

\title{
An edge-based unstructured mesh framework for atmospheric flows
}

PLEASE CITE THE PUBLISHED VERSION

http://dx.doi.org/10.1016/j.compfluid.2010.10.020

PUBLISHER

(c) Elsevier

VERSION

AM (Accepted Manuscript)

LICENCE

CC BY-NC-ND 4.0

REPOSITORY RECORD

Szmelter, Joanna, and Piotr K. Smolarkiewicz. 2019. "An Edge-based Unstructured Mesh Framework for Atmospheric Flows”. figshare. https://hdl.handle.net/2134/13368. 
This item was submitted to Loughborough's Institutional Repository (https://dspace.lboro.ac.uk/) by the author and is made available under the following Creative Commons Licence conditions.

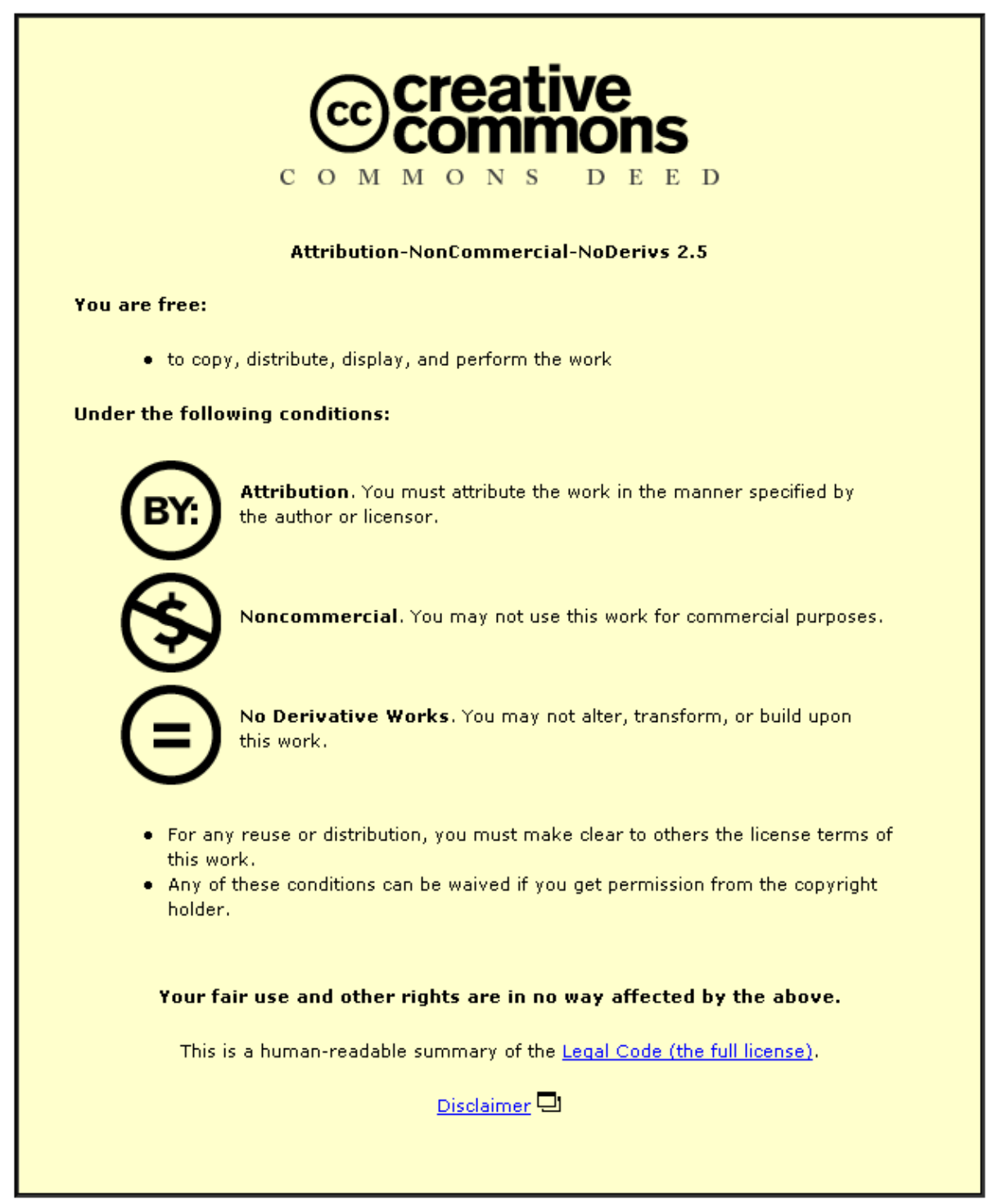

For the full text of this licence, please go to: http://creativecommons.org/licenses/by-nc-nd/2.5/ 


\title{
An Edge Based Unstructured Mesh Framework for Atmospheric Flows
}

\author{
Joanna Szmelter ${ }^{\mathrm{a}}$, Piotr K. Smolarkiewicz ${ }^{\mathrm{b}}$ \\ ${ }^{a}$ Loughborough University, Leicestershire, LE11 3TU, UK \\ ${ }^{b}$ National Center for Atmospheric Research, Boulder, CO 80307, USA
}

\begin{abstract}
This paper describes an unstructured/hybrid mesh framework providing a robust environment for multiscale atmospheric modeling. The framework builds on nonoscillatory forward-in-time MPDATA solvers using finite volume edge based discretization, and admits meshes with arbitrarily shaped cells. The numerical formulation is equally applicable to global and limited area models. Theoretical considerations are supported with canonical examples of slab-symmetric, nonhydrostatic orographic problems in weakly and strongly stratified flow regimes and three-dimensional hydrostatic analogues of the strongly stratified case on a slowly and rapidly rotating sphere.

Keywords: unstructured meshes, atmospheric waves, geospherical framework, MPDATA, nonoscillatory differencing
\end{abstract}

\section{Introduction}

The higher resolutions necessary to capture detailed flow features in multiscale geophysical flows are not available in routinely used Cartesian-grid models, because they would require a many-fold increase in the number of computational points. The rigid connectivity of structured grids used in 
the simulation of atmospheric/oceanic circulations (viz. rotating stratified flows) imposes severe limitations on mesh adaptivity to flow features or the complex geometry of physical domains. In contrast, for many problems, a wide range of scales in atmospheric flows, heterogeneous distribution of regions of interest, or complex geometry can be accommodated efficiently with fully unstructured mesh technology. The realization of limitations of structured grids versus potential benefits of flexible meshing has stimulated recent interest within the atmospheric science community in the development of unstructured mesh solvers; see [1] and references therein for reviews.

Studies exploring modeling of atmospheric flows on unstructured meshes date back to the nineteen sixties [2], but the arguments for flexible mesh adaptivity have emerged more recently (cf. the collection of papers in the special issue [3]) with the advent of multiscale Earth-system modeling and climate prediction. However, as yet, adaptive-mesh atmospheric models have not met the demands of the modern operational weather prediction and climate studies, reviewed in the collection of works [4].

Even though the operational efficacy of unstructured meshes is yet to be proven, Earth-system modeling invokes research activities in a broad range of processes and phenomena, the computational study of which may benefit from capabilities of flexible meshing. One such important research area is the modeling of inertia-gravity waves. These waves are ubiquitous in the atmosphere and oceans, evince the abundance of multiscale phenomena [5], and are consequential for weather and climate [6]. Their occurrence and form depend on a relative magnitude and structure of ambient flow, density/entropy stratification and forcing. 
To date, research into unstructured mesh atmospheric models was largely confined to idealized applications addressing either synoptic flows in the lowest order long-wave approximation governed by the shallow water equations, or small-scale buoyant phenomena occurring in a neutrally stratified quiescent atmosphere - prototypes of natural convection and density currents. Compared to these two diverse classes of motions, applications addressing the dynamics of internal inertia-gravity waves are scarce. In particular, the authors are unaware of any published fully unstructured mesh solutions emulating the theory of mountain waves. Because of their intricacy, such solutions constitute canonical benchmarks for numerical weather prediction codes. Here we demonstrate original solutions for two such benchmarks, in two and three spatial dimensions for nonhydrostatic and primitive hydrostatic equations, respectively, thus substantiating the potential of unstructured meshes for simulation of the inertia-gravity wave dynamics.

This paper presents an algorithmic framework suitable for the development of all-scale atmospheric flow unstructured/hybrid mesh models. The proposed approach generalizes the methodologies proven in the structured grid computational model EULAG for simulating thermo-fluid flows across a wide range of scales and physical scenarios; see [7] for a review and comprehensive list of references. The key elements of the framework include the median-dual finite volume edge-based [8], nonoscillatory advection schemes MPDATA [9]; a robust nonsymmetric Krylov-subspace elliptic solver [10]; and a class of nonoscillatory forward-in-time (NFT) algorithms for integrating governing PDEs. The NFT algorithms, reviewed in [7], have been already utilized in the authors' recent developments of unstructured mesh solvers for 
gas dynamics [11]. Herein, the unstructured mesh NFT framework is employed to derive a new model for stratified orographic flows.

The structured grid NFT framework is formulated in generalized timedependent curvilinear coordinates, enabling dynamic grid adaptivity via continuous mappings in either Cartesian or spherical domains [7]. Unconventionally for flexible mesh models, the unstructured mesh NFT framework is also formulated in curvilinear coordinates. In particular, this is useful for modeling global circulations in spherical geometry [1] employing a classical geospherical reference frame with the governing equations cast in the latitudelongitude surface-based coordinates (section 7.2 in [12]). While retaining the benefits of the classical formulation, common in theoretical geo/astro physics, its notorious limitations associated with the convergence of meridians in the polar regions are circumvented by exploiting the flexibility of unstructured meshes. The latter is highlighted in Figure 1, which shows two alternate views of the mesh employed in simulations of global rotating stratified flows past an isolated mountain discussed in section 4 .
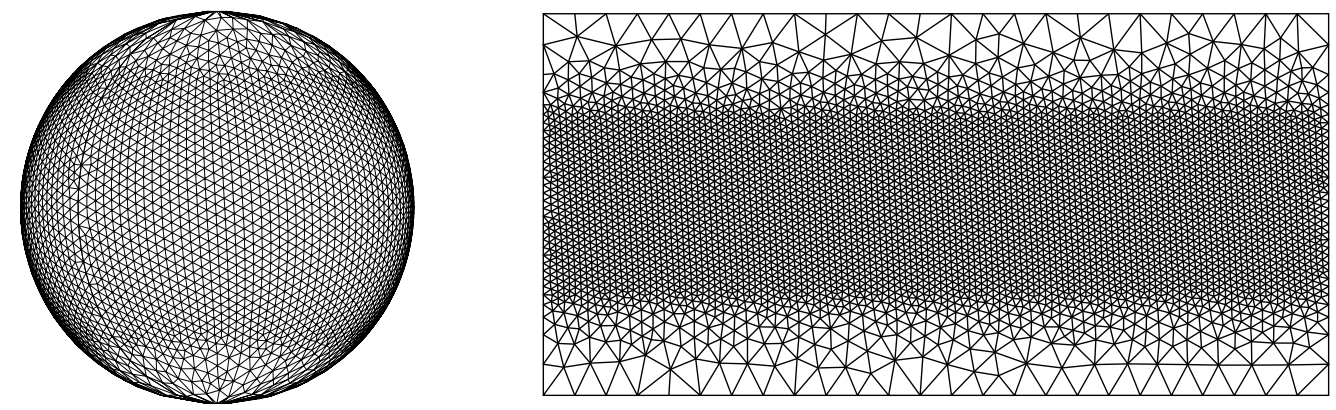

Figure 1: Triangular mesh in the physical space on a sphere and in a transformed (computational) latitude-longitude domain underlying the geospherical framework 


\section{A general NFT unstructured mesh framework}

The NFT methods had been put forward in the early nineties in the context of geophysical flows and structured grid solvers [13]. NFT labels a class of second-order-accurate, either semi-Lagrangian (trajectory wise) or Eulerian (finite-volume wise), algorithms that rely on the strengths of two-timelevel nonlinear advection techniques that suppress/reduce/control numerical oscillations characteristic of higher-order linear schemes. The underlying theory derives from the truncation-error analysis of the forward-in-time approximations [13] for an archetype system of inhomogeneous PDEs for fluids

$$
\frac{\partial G \boldsymbol{\Phi}}{\partial t}+\nabla \cdot(\mathbf{V} \boldsymbol{\Phi})=G \boldsymbol{R}
$$

in abstraction from any particular assumptions on the nature of the vector of conservative dependent variables $\boldsymbol{\Phi}$ and the associated vector of the right-hand-side (rhs) forcings $\boldsymbol{R}$, but driven solely by the requirement of the second-order accuracy for an arbitrary advective velocity $\mathbf{V}(\mathbf{x}, t):=G \dot{\mathbf{x}}$. Here, $G \equiv\left|g_{p q}\right|^{1 / 2}$ is the Jacobian defined in terms of the metric tensor $g_{p q}$ of a curvilinear coordinate system $\mathbf{x}=\left(x^{1}, x^{2}, x^{3}\right) \equiv(x, y, z)$ with the fundamental metric form $d s^{2}=g_{p q} d x^{p} d x^{q}$, so $\dot{\mathbf{x}}$ is the contravariant velocity in

the curvilinear coordinate frame; see [14] for an exposition. The resulting template algorithm for (1) takes a compact functional form

$$
\boldsymbol{\Phi}_{i}^{n+1}=\mathcal{A}_{i}\left(\boldsymbol{\Phi}^{n}+0.5 \delta t \boldsymbol{\mathcal { R }}^{n}, \mathbf{V}^{n+1 / 2}, G\right)+0.5 \delta t \boldsymbol{\mathcal { R }}_{i}^{n+1} \equiv \widehat{\boldsymbol{\Phi}}_{i}+0.5 \delta t \boldsymbol{\mathcal { R }}_{i}^{n+1},
$$

where $n$ and $i$ refer to the temporal and spatial position on the mesh, $\mathcal{A}$ symbolizes a forward-in-time nonoscillatory transport operator (viz. advection scheme) assumed second-order accurate for a homogeneous case of (1) 
with time-independent $\mathbf{V}$. Furthermore, $\mathbf{V}^{n+1 / 2}$ is an $\mathcal{O}\left(\delta t^{2}\right)$ estimate of $\mathbf{V}$ at $t+0.5 \delta t$, and $\boldsymbol{R}^{n+1}=\boldsymbol{R}(t+\delta t)+\boldsymbol{O}\left(\delta t^{3}\right)$ can either be an explicit estimate (viz. predictor) or depend implicitly on the components of $\boldsymbol{\Phi}$.

In NFT schemes there are two elements defining the solver: i) the choice of the transport operator $\mathcal{A}$; and ii) the approach for evaluating $\boldsymbol{\mathcal { R }}^{n+1}$ on the rhs. For the former, our method of choice is MPDATA [9], for its genuine multidimensionality free of splitting errors, easy accuracy-sustaining generalization to unstructured meshes, and suitability for DNS, LES, and ILES. In general, the evaluation of $\boldsymbol{R}^{n+1}$ depends on the physical problem, the particular form of governing PDEs and a selection of dependent variables. Two diverse implementations of the outlined abstract framework will be presented in the following sections.

\section{A local area nonhydrostatic model}

First, we illustrate how the approach can be used for the development of local area nonhydrostatic models. Here, the unstructured mesh NFT framework is applied in an $x z$ Cartesian plane with the metric tensor $g_{p q}=\delta_{p q}$, upon which $G \equiv 1$ in (1) and (2). The benchmark problem addressed is a stratified ambient flow with a constant buoyancy frequency $N=1 \mathrm{~s}^{-1}$ and uniform wind $\mathbf{V}_{0}=\left(U_{0}, 0\right), U_{0}=10 \mathrm{~ms}^{-1}$, impinging on an isolated ridge of the form $h(x)=h_{0}\left[1+(x / L)^{2}\right]^{-1}$ centered at the origin of the $[-16.7 L, 25 L] \times[0,25 L]$ domain. The hill's half-width $L$ is fixed, whereas the height $h_{0}=0.25 L$ or $h_{0}=0.5 L$. The respective Froude numbers, $F r=U_{0} / N h_{0}$, are $F r=1.66$ or $F r=0.83$ indicating a weakly- and strongly-nonlinear gravity-wave responses [15]. The problem is nonhydro- 
static, because $N L / U_{0}=2.4$; that is, the horizontal scale of the problem is comparable to the asymptotic wavelength $\lambda_{0} \approx 2 \pi U_{0} / N$ of the induced mountain wave. The solutions are obtained by the canonical incompressible Boussinesq model [5], for which the governing conservation laws of mass, momentum and thermodynamic properties take the form

$$
\begin{aligned}
& \nabla \cdot\left(\mathbf{V} \rho_{o}\right)=0 \\
& \frac{\partial \rho_{o} V^{I}}{\partial t}+\nabla \cdot\left(\mathbf{V} \rho_{o} V^{I}\right)=-\rho_{o} \frac{\partial \widetilde{p}}{\partial x^{I}}+\rho_{o} g \frac{\theta^{\prime}}{\theta_{o}} \delta_{I 3} \\
& \frac{\partial \rho_{o} \theta}{\partial t}+\nabla \cdot\left(\mathbf{V} \rho_{o} \theta\right)=0 .
\end{aligned}
$$

Here, $\rho$ and $\theta$ denote the density and potential temperature ${ }^{1}, V^{I}(I=1$ and $I=3$ ) refers to the velocity components in the horizontal and the vertical, and $\widetilde{p}=p^{\prime} / \rho_{o}$. Subscripts ${ }_{o}$ denote constant values of the static reference state, while primes denote perturbations with respect to the static ambient state characterized by constant stratification $S_{o}=N^{2} / g$, with the acceleration of gravity $g=9.81 \mathrm{~ms}^{-2}$. The lower boundary is impermeable, whereas at the top and the lateral boundaries $\left(\mathbf{V}-\mathbf{V}_{0}\right) \cdot \mathbf{n}=0$ is assumed; $\mathbf{n}$ is the outward unit normal to the model boundaries. The $2 \lambda_{0}$-thick absorbing layers adjacent to the top and lateral boundaries mitigate wave reflections, thus mimicking open boundary conditions. The initial flow is potential, $\mathbf{V}(\mathbf{x}, t=0)=\mathbf{V}_{0}-\nabla \phi$, determined by mass continuity and the boundary conditions.

The execution of the template algorithm (2) for (3) proceeds in three distinct stages. First, the estimate of the advective velocity at $t+0.5 \delta t$ is

\footnotetext{
${ }^{1}$ The potential temperature in (3) amounts to the specific entropy, because $s=c_{p} \ln \theta$; $c_{p}$ is the specific heat at constant pressure.
} 
evaluated using, in general, either a linear extrapolation in time or a firstorder predictor. ${ }^{2}$ Second, advecting the auxiliary fields $\Phi^{n}+0.5 \delta t \mathcal{R}^{n}$ for the momentum components and $\theta$ provides the respective $\widehat{\boldsymbol{\Phi}}$ counterparts of (2). For adiabatic dynamics considered here, this stage already completes the solution of the entropy equation and provides an $\mathcal{O}\left(\delta t^{3}\right)$ estimate of the buoyancy force on the rhs of the vertical momentum equation. In consequence, in the corresponding realizations of (2) for momenta

$$
\rho_{o} \mathbf{V}_{i}^{n+1}={\widehat{\rho_{o} \mathbf{V}_{i}}}_{i}-0.5 \delta t\left(\rho_{o} \nabla \widetilde{p}-\rho_{o} g \frac{\theta^{\prime}}{\theta_{o}} \mathbf{k}\right)_{i}^{n+1}
$$

the components of the pressure gradient $\nabla \widetilde{p}^{n+1}$ are the only unknowns on the rhs; here $\mathbf{k}=\nabla z$ denotes the unit vector in the vertical. The third stage of the solution procedure consists of formulating and inverting a sparse linear problem for pressure. Acting with the discrete divergence operator on (4), and noting that $\nabla_{i} \cdot\left(\rho_{o} \mathbf{V}\right)^{n+1}$ must vanish on the left-hand-side due to the continuity constraint in (3), implies the discretized elliptic pressure equation

$$
\forall_{i} \quad \nabla_{i} \cdot\left(\widetilde{\rho_{o} \mathbf{V}}-0.5 \delta t \rho_{o} \nabla \widetilde{p}^{n+1}\right)=0
$$

where $\widetilde{\rho_{o} \mathbf{V}}=\widehat{\rho_{o} \mathbf{V}}+0.5 \delta \operatorname{tg} \rho_{o}\left(\theta^{\prime} / \theta_{o}\right)^{n+1} \mathbf{k}$ forms the explicit part of the problem. The resulting system (5) is solved to a specified tolerance using the preconditioned generalized conjugate residual algorithm, broadly discussed in [10] and references therein. With the selected model setups, the integrability condition for $(5), \oint \rho_{o} \mathbf{V}^{n+1} \cdot \mathbf{n}=0$, is satisfied to machine precision.

\footnotetext{
${ }^{2}$ Here, we use exclusively the advective (nonconservative) centered-in-space Eulerforward predictor with an implicit pressure-correction leading to the associated elliptic problem, required to assure nondivergent solutions.
} 
Notably, Dirichlet boundary conditions for $\mathbf{V}^{n+1}$ imply Neumann conditions for pressure $\widetilde{p}^{n+1}$ via $\left(\widetilde{\rho_{o} \mathbf{V}}-0.5 \delta t \rho_{o} \nabla \widetilde{p}^{n+1}\right) \cdot \mathbf{n}=\mathbf{V}_{0} \cdot \mathbf{n}$. Given the solution to (5), combining the updated buoyancy and pressure-gradient components on the rhs of (4) completes the solution.
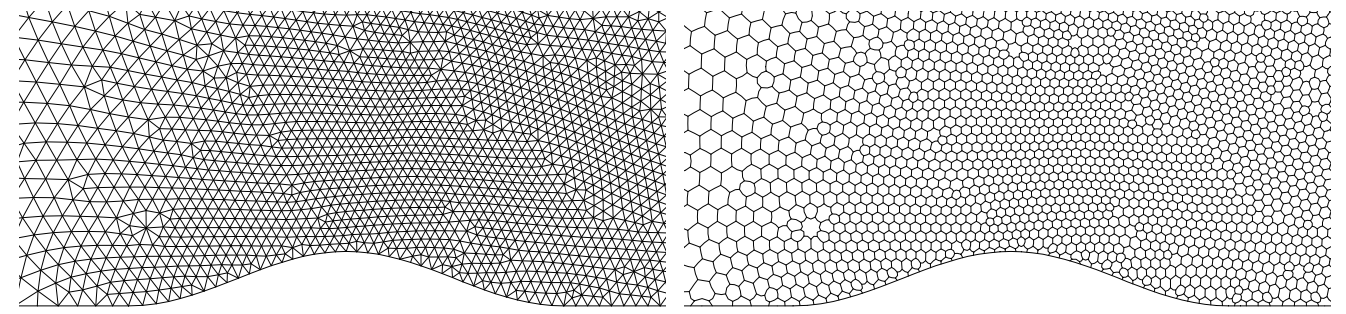

Figure 2: Details of mesh in the vicinity of the hill; edges of the triangular mesh (left) and the polygonal median-dual finite-volume mesh (right).

The derivation of MPDATA and discrete differential operators in the edge-based median-dual finite-volume approach used here are detailed in $[9,16]$. This spatial discretization approach is illustrated in Figure 2. The edges in the left panel connect nodes of a triangular mesh and pierce faces of median-dual cells (in the right panel) surrounding each node. In the two examples considered in this section triangular meshes with approximately 39,500 points were refined to represent both the hill geometry and the main portion of the wave train. The minimum nodal spacing of $L / 12$ was prescribed in the middle of the hill's base and was gradually reduced with altitude to $L / 8$ spacing following the main wave train. The nodal spacing was also smoothly reduced in the upwind and downwind directions (away from the main portion of the wave train) to $5 L / 3$ and $L / 2$, respectively.

Figure 3 highlights the model solutions for the weakly- and stronglynonlinear responses by showing the isentropes in a developing flow after di- 

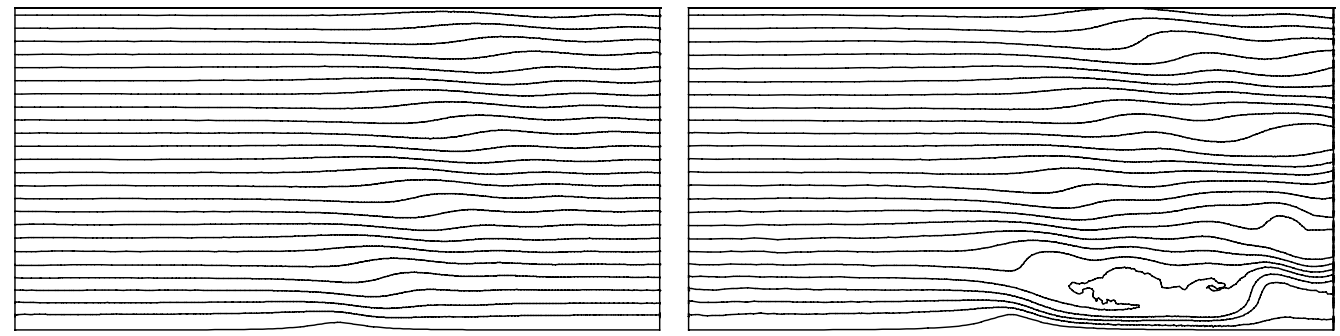

Figure 3: Isentropes simulated using the two-dimensional nonhydrostatic model; $F r \lesssim 2$ and $F r \lesssim 1$, in the left and right plate, respectively.

mensionless time $\tau=t U_{0} / L=150 .^{3}$ In the former case, the mountain wave propagates at an angle $\alpha \approx 60^{\circ}$ off the horizontal - measured from the corresponding contour plot of the vertical velocity (not shown) — consistent with the linear steady-state prediction $\alpha=\tan ^{-1} \sqrt{\left(U_{0} / L N\right)^{2}-1}$; section 2.2.3 in [17]. In contrast, for $F r \underset{\sim}{<1}$ the lee wave breaking and strong downslope winds (evidenced by isentrope compression) with the turbulent flow aloft are indicative of much studied wind-storm phenomena in mountainous terrain [6]. Both solutions were compared with similar solutions generated with structured grid EULAG model, using a uniform spacing of $\approx L / 12$ covering identical domain with 157,184 grid points. Regardless of the fundamental differences in the spatial discretization and minor (for this problem) discrepancies in the time integration schemes, ${ }^{4}$ the two model solutions are hardly distinguishable in figures for the weakly nonlinear case and differ merely in

\footnotetext{
${ }^{3}$ This equals 2.5 time periods required for the wave energy of the dominant horizontal mode with the wavenumber $k=1 / L$ to propagate over the model depth.

${ }^{4}$ While EULAG uses a fully implicit trapezoidal integration of the gravity-wave forcing terms and boundary absorbers [7], the unstructured mesh model still represents these terms explicitly.
} 
physically insignificant details for $F r \lesssim 1$; not shown. For substantiation, consider that in the weakly-nonlinear case the structured grid and unstructured mesh solutions both differ from the linear theory estimates by no more than $\approx 3 \%$ in the wavelength $\lambda_{0}$ and by no more than $\approx 8 \%$ in the propagation angle $\alpha$. Furthermore, they both show the same distribution of the wave amplitude with height, with an average per wavelength (over seven wavelenghts) loss of $\approx 7 \%$ attributed primarily to the dispersive character of the nonhydrostatic mountain wave; cf. section 2.2 .3 in [17].

\section{A global hydrostatic model}

The second application of the unstructured mesh NFT framework involves global hydrostatic flows with the governing PDEs cast in orthogonal latitudelongitude $(\varphi, \lambda)$ surface-based coordinates, viz. the classical geospherical reference frame rooted in the differential calculus on manifolds, see [1] for a discussion. Here, the fundamental metric form $d s^{2}=g_{11} d x^{1} d x^{1}+g_{22} d x^{2} d x^{2} \equiv$ $(a \cos \varphi d \lambda)^{2}+(a d \varphi)^{2} \equiv\left(h_{x} d x\right)^{2}+\left(h_{y} d y\right)^{2}$, where $a$ denotes the sphere's radius. The triple notation and the map factors $h_{x}:=\sqrt{g_{11}}=a \cos \varphi$ and $h_{y}:=\sqrt{g_{22}}=a$ are introduced to: i) bridge a formal tensor notation valid for generalized time-dependent curvilinear coordinates [14] with the simplified notation for orthogonal systems common in the meteorological literature (e.g., section 2.6 in [18]); and ii) relate both these notations to the vector symbolism of the algorithmic framework (1) and (2). Consequently, the Jacobian $G$, the Nabla operator $\nabla$, and the advective velocity $\mathbf{V}$ in (1) and (2) become $G=h_{x} h_{y}, \nabla=(\partial / \partial \lambda, \partial / \partial \varphi)$, and $\mathbf{V}=(G \dot{\lambda}, G \dot{\varphi})$, respectively.

The particular set of the PDEs adopted describes rotating stratified flu- 
ids under the shallow atmosphere approximation, cast in a hybrid EulerianLagrangian form with a material coordinate $\zeta$ monotonically increasing with height (section 7.4 in [12]):

$$
\begin{aligned}
\frac{\partial G \mathcal{D}}{\partial t}+\nabla \cdot(\mathbf{V D}) & =0 \\
\frac{\partial G Q_{x}}{\partial t}+\nabla \cdot\left(\mathbf{V} Q_{x}\right) & =G\left(-\frac{1}{h_{x}} \mathcal{D} \frac{\partial M}{\partial x}+f Q_{y}-\frac{1}{G \mathcal{D}} \frac{\partial h_{x}}{\partial y} Q_{x} Q_{y}\right) \\
\frac{\partial G Q_{y}}{\partial t}+\nabla \cdot\left(\mathbf{V} Q_{y}\right) & =G\left(-\frac{1}{h_{y}} \mathcal{D} \frac{\partial M}{\partial y}-f Q_{x}+\frac{1}{G \mathcal{D}} \frac{\partial h_{x}}{\partial y} Q_{x}^{2}\right) \\
\frac{\partial M}{\partial \zeta}=\Pi &
\end{aligned}
$$

Here the position vector $\mathbf{x}=(x, y, \zeta)$, and $\mathcal{D} \equiv \partial p / \partial \zeta$ may be viewed as a generalized mass density. The momentum vector $\mathbf{Q}=\left(Q_{x}, Q_{y}\right)=\mathcal{D} \mathbf{v}$, where $\mathbf{v}$ is a physical velocity (with dimensions of length/time) measurable in a local Cartesian frame tangent to the sphere's surface and related to the contravariant and the advective velocity via

$$
\left(v_{x}, v_{y}\right)=\left(h_{x} \dot{\lambda}, h_{y} \dot{\varphi}\right)=\left(V_{x} h_{y}^{-1}, V_{y} h_{x}^{-1}\right)
$$

On the rhs of the momentum equations an equivalent of the pressure gradient force depends on the Montgomery potential (or Bernoulli function) $M=$ $g H+\zeta \Pi$, where $H$ is the height of a material surface, and $\Pi$ is a transform of the pressure $p$. An isentropic model [19] is used in the calculations presented here, hence $\zeta=\theta$ and $\Pi=c_{p}\left(p / p_{o}\right)^{R_{d} / c_{p}}$ is the Exner function; wherein $R_{d}$ is the gas constant for dry air, and $p_{o}$ is a reference pressure. The remaining terms on the rhs include the Coriolis and the metric forces, with $f=2 \Omega \sin \varphi$ and $\Omega$ denoting the planetary rotation rate.

Although the system (6) governs continuously stratified fluids, its discretization in $\zeta$ leads to a system resembling a stack of shallow-water layers 
[1] coupled via the hydrostatic relation $\partial M / \partial \zeta=\Pi$. Because the Lagrangiangrid interval $\delta_{k} \zeta=\zeta_{k+1}-\zeta_{k}$ is fixed for each $k=1, \ldots, K$ layer, the pressure thickness $\delta_{k} p:=p_{k+1}-p_{k}$ becomes the actual discrete variable in lieu of $\mathcal{D}$. The continuity and momentum equations in (6) adhere to the form of an archetype PDE (1), and their integration adopts the template algorithm (2). The implementation of (2) in the hybrid Eulerian-Lagrangian system (6) is detailed in [1]. Below we only summarize the three distinct stages of the model algorithm.

Having predicted the advective velocities $\mathbf{V}^{n+1 / 2}$ by integrating to $\mathcal{O}\left(\delta t^{2}\right)$ the evolutionary form of the velocity equation $\mathcal{D} d \mathbf{v} / d t=\mathbf{R}$ (section 3.1 in [1]), the $\widehat{\Phi}_{i}$ on the rhs of (2) representing advection of $\delta p, Q_{x}$ and $Q_{y}$ augmented with the contributions from their respective forcings are evaluated independently for all $K$ layers. Due to the homogeneity of the continuity equation, this stage instantly provides the updated pressure thickness $\delta_{k} p_{i}^{n+1}$ of each control volume $\mathcal{V}_{i k}^{n+1}=G_{i} \mathcal{V}_{i}^{*} \delta_{k} p_{i}^{n+1} ; \mathcal{V}_{i}^{*}$ is the planar area of the horizontal cell containing node $i$ [1]. Next, assuming a free-surface boundary condition aloft, the pressure $p$ is recovered recurrently (downward) from its definition, independently for all horizontal nodes $i$. Transforming $p$ into the Exner function, successive upward integration of the hydrostatic relation $\partial M^{n+1} / \partial \zeta=\Pi^{n+1}$ provides the values of $M^{n+1}$ for all discrete values of $\zeta$. Finally, independent for all $\zeta$, the gradient force at $t^{n+1}$ is evaluated explicitly from $M^{n+1}$ and $\delta_{k} p^{n+1}$, whereupon the momenta are evaluated iteratively, locally in all nodes $i$, assuming implicit representation of the Coriolis force, with the metric forces lagged (section 3.1 in [1]). The updated momenta and pressure thickness provide the physical velocity update, whereupon the 
contravariant velocity required to predict the advective velocity for the subsequent time step is diagnosed from (7).

To illustrate the applicability of the algorithmic framework across a broad range of scales we consider two cases of strongly-stratified and rotating 3D flow past an isolated hill. The first case, after [1], simulates the mesoscale response by reducing the planet's radius hundredfold while keeping the Earth rotation fixed [20]. In the second case the rotation rate is also increased hundredfold, thus simulating the synoptic-scale response. The analytic axisymmetric hill is placed at the equator, and its height decays as $h(x, y)=$ $h_{0}\left[1+(l / L)^{2}\right]^{-3 / 2}$, where $l(x, y)$ denotes the distance from the center of the hill on the spherical surface. The height and half-width are $L=12.4 \cdot 10^{3} \mathrm{~m}$ and $h_{0}=500 \mathrm{~m}$, respectively, and the geostrophically balanced zonal ambient flow $\mathbf{v}_{e}(\varphi)=\left(U_{0} \cos \varphi, 0\right)$ forms the initial condition, with $U_{0}=10 \mathrm{~ms}^{-1}$. In both calculations $F r=0.5$, whereas the Rossby number $R_{o}=U_{0} / L f$ is $R_{o} \gg 1$ and $R_{o} \gtrsim 1$, respectively, for the meso- and the synoptic-scale response. ${ }^{5}$ The triangular mesh corresponding to the computational dual mesh employed is shown in Figure 1; for the display of the mesh following the hill along the lowest model level, see Figure 15 in [1].

Figure 4 shows the instantaneous distribution of the isentropes (i.e., the instantaneous values of the model material coordinate in the vertical) in the equatorial $x z$ cross-section after four hours of the simulated time. Figure 5 shows the concomitant displacements of the isentropic surfaces with the undisturbed equatorial height $H(\lambda, \varphi=0, t=0)=,0.25 \pi U_{0} N^{-1}$ (i.e.,

\footnotetext{
${ }^{5}$ Because $f=0$ at the equator but the results depend on the rotation rate, $f=$ $2 \Omega \sin (L / a)$ is used as a more representative value.
} 
the eight of the dominant vertical wavelength $\lambda_{0}$ at $R_{o} \gg 1$ ) together with the flow vectors on these surfaces. ${ }^{6}$ Concurrently, Figures 4 and 5 illustrate salient flow features.
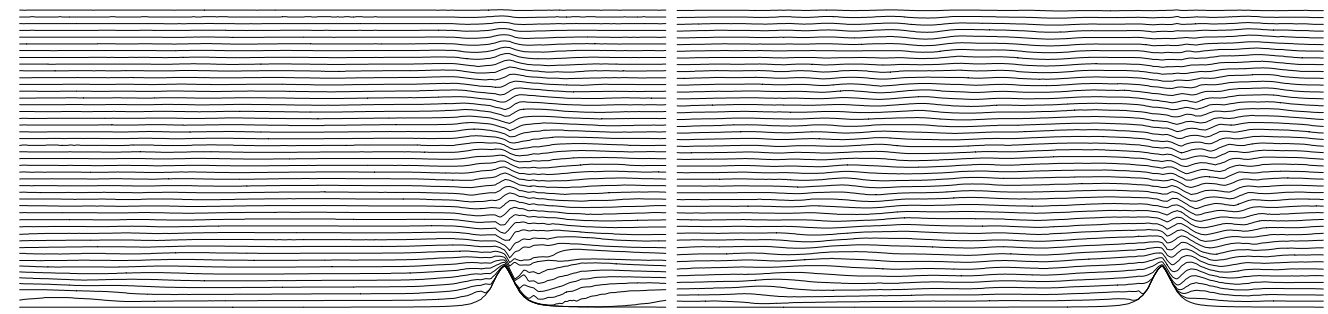

Figure 4: Isentropes in the equatorial-vertical plane, simulated using the 3D hydrostatic model for global orographic flow with $F r=0.5 ; R o \gg 1$ (left), and $R o \gtrsim 1$ (right).
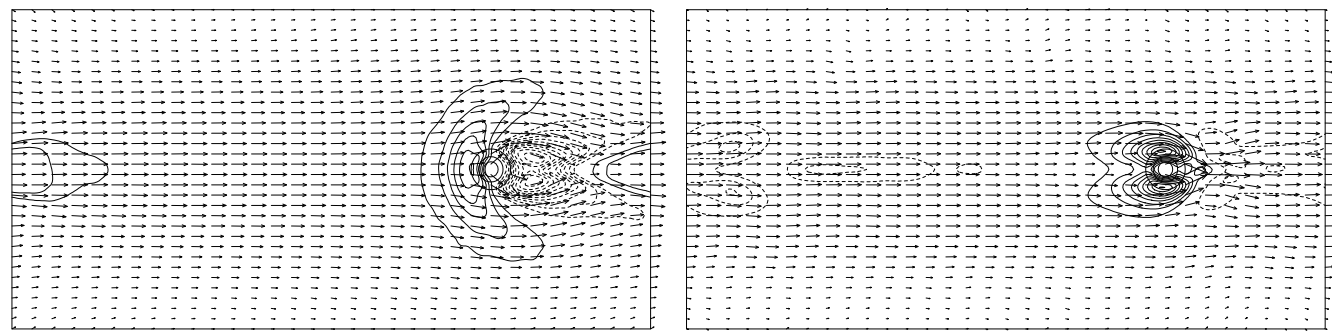

Figure 5: As in Figure 4 but for vertical displacements of, and superimposed flow vectors at, the isentropic surface with the undisturbed equatorial height $\approx 0.25 \pi U_{0} N^{-1}$; contours of the hill height are also superimposed.

For the slowly-rotating, strongly-stratified case (left panels) — already discussed in [1] for the analogous isopycnic model - the results evince flow blocking on the lower upwind side of the hill and intense lee eddies, characteristic of low Froude number 3D mesoscale flows widely studied in laboratory and numerical simulation [21]. For the hundredfold faster rotation $R_{o} \approx 5$,

\footnotetext{
${ }^{6}$ For visualization, the flow vectors were interpolated to a Cartesian grid.
} 
the Rossby deformation radius $L_{R}=h_{o} N / f \approx 13 \cdot 10^{3} \mathrm{~m}$ becomes comparable to $L$, upon which the effects due to the rotation and stratification occur on similar horizontal scales while counteracting each other. The planetary rotation produces strong uplift of the isentropes on the mountain lateral sides and compensates the vorticity of the lee eddies, whereas aloft the mountain wave disperses with altitude. These effects are consistent with theoretical predictions $[17,21]$, and with the equivalent EULAG solutions (not shown) on the $128 \times 64 \times 91$ grid. Noteworthy, the unstructured mesh shown in Figure 1 (repeated at 91 isentropic levels) consists of 4532 nodes, thus effecting in about twice smaller computational problem then in EULAG. Insofar as the economy of computations is concerned, the differences between the two models preclude a rigorous comparison. ${ }^{7}$ Yet contrasting the EULAG integration wall-clock time $\approx 9$ min using 32 processors of IBM Power 575 supercomputer with $\approx 70$ min execution of the unstructured mesh model using a single laptop processor illustrates the potential of unstructured meshes for a cost-effective simulation of atmospheric flows.

\section{Remarks}

Herein, a two-dimensional nonhydrostatic model build on an edge-based unstructured mesh NFT framework is introduced. Canonical benchmarks demonstrate the suitability of flexible meshing for modeling atmospheric inertia-gravity waves forming a constitutive element of weather and climate. Examination of the results against theoretical estimates and respective re-

\footnotetext{
${ }^{7}$ EULAG integrates the nonhydrostatic anelastic equations free of the fast external mode, but the unstructured-mesh hydrostatic model is free of a costly Poisson solver.
} 
sults obtained with an established structured grid model corroborates our earlier conclusions $[9,11]$ that the edge-based discretization sustains the accuracy of structured grid NFT schemes. Furthermore, the results obtained with a global hydrostatic model demonstrate that the benefits of the classical geospherical formulation, used in the majority of established weather and climate models employing either the finite-difference or spectral methods, also hold for unstructured meshes. The unstructured mesh developments retain proven properties of the NFT MPDATA structured grid solvers [7] — the advocated algorithms are fully multidimensional, rigorously sign preserving and nonlinearly stable. The NFT framework is applicable to arbitrarily shaped hybrid meshes that allow for investigating optimal mesh-point distributions $[9,16,11,1]$. A flexible use of various mesh adaptivity techniques, with an explicit analytic form of the error estimator naturally arising from MPDATA is also possible and has already been demonstrated for compressible flows [16].

\section{Acknowledgments}

A personal review by Robert Sharman is gratefully acknowledged. This work was supported in part by the the NERC award NE/G004358 and DOE award DE-FG02-08ER64535. The National Center for Atmospheric Research is sponsored by the National Science Foundation.

\section{References}

[1] J. Szmelter and P.K. Smolarkiewicz, An edge based unstructured mesh discretization in geospherical framework, J. Comput. Phys., 229, 4980-4995 (2010). 
[2] D.L. Williamson, The evolution of dynamical cores for global atmospheric models, J. Meteor. Soc. Japan, 85B, 241-268 (2008).

[3] Theme Issue 'Mesh generation and mesh adaptation for large-scale Earthsystem modelling' compiled and edited by N. Nikiforakis, Phil. Trans. R. Soc. A, 367, 4473-4654 (2009).

[4] Special Issue 'Predicting weather, climate and extreme events' Guest Editors M.J. Miller and P.K. Smolarkiewicz, J. Comput. Phys., 227, 3429-3730 (2008).

[5] M.G. Wurtele, R.D. Sharman and A. Datta, Atmospheric lee waves, Annu. Rev. Fluid. Mech., 28, 429-476 (1996).

[6] J.D. Doyle, D.R. Durran, C. Chen, et al., An intercomparison of modelpredicted wave breaking for the 11 January 1972 Boulder Windstorm, Mon Weather Rev., 128, 901-914 (2000).

[7] J.M. Prusa, P.K. Smolarkiewicz and A.A Wyszogrodzki, EULAG, a computational model for multiscale flows, Comput. Fluids, 37, 1193-1207 (2008).

[8] Barth TJ. Aspects of unstructured grids and finite volume solvers for the Euler and Navier-Stokes equations. In Special Course on Unstructured Grid Methods for Advection Dominated Flows AGARD Report 787; 1992:6.1-6.61.

[9] P.K. Smolarkiewicz and J. Szmelter, MPDATA: An edge-based unstructuredgrid formulation, J. Comput. Phys., 206, 624-649 (2005).

[10] P.K. Smolarkiewicz, C. Temperton, S.J. Thomas, and A.A. Wyszogrodzki. Spectral Preconditioners for nonhydrostatic atmospheric models: extreme applications. Proceedings of the ECMWF Seminar Series on Recent developments in numerical methods for atmospheric and ocean modelling, Reading, UK, 203-220 (2004). 
[11] P.K. Smolarkiewicz and J. Szmelter, Iterated Upwind Schemes for Gas Dynamics, J. Comput. Phys., 228, 33-54 (2009).

[12] Dutton J.A. The Ceaseless Wind. Dover Publications, 1986, pp. 617.

[13] P.K. Smolarkiewicz and L.G. Margolin, On forward-in-time differencing for fluids: extension to a curvilinear framework, Monthly Weather Rev., 121, 1847-1859 (1993).

[14] J.M. Prusa, P.K. Smolarkiewicz, An all-scale anelastic model for geophysical flows: dynamic grid deformation, J. Comput. Phys., 190, 601-622 (2003).

[15] D.R. Durran, Lee waves and mountain waves, in Encyclopedia of Atmospheric Sciences, Holton J.R., J. Pyle and J.A. Curry, eds., Elsevier Sciences Ltd., 1161-1169 (2003).

[16] J. Szmelter and P.K. Smolarkiewicz, MPDATA error estimator for mesh adaptivity, Int. J. Numer. Methods Fluids, 50, 1269-1293 (2006).

[17] R.B. Smith, The influence of the mountains on the atmosphere, Advances in Geophysics, 21, 87-230 (1979), Academic Press.

[18] D.L. Williamson, J.B. Drake, J.J. Hack, R. Jakob, P.N. Swarztrauber, A Standard Test Set for Numerical Approximations to the Shallow Water Equations in Spherical Geometry, J. Comput. Phys., 102, 211-224 (1992).

[19] J.M. Reisner and P.K. Smolarkiewicz, Thermally forced low Froude number flow past three-dimensional obstacles, J. Atmos. Sci., 51, 117-133 (1994).

[20] N.P. Wedi and P.K. Smolarkiewicz, A Framework for testing global nonhydrostatic models, Q.J. Roy. Meteorol. Soc., 135, 469-484 (2009).

[21] J.C.R. Hunt, H. Olafsson and P. Bougeault, Coriolis effects on orographic and mesoscale flows, Q. J. R. Meteorol. Soc., 127 601-633 (2001). 